\title{
Comparative evaluation of ten lateral flow immunoassays to
}

\section{detect SARS-CoV-2 antibodies [version 1; peer review: awaiting}

\section{peer review]}

\author{
Gala Garrod 1*, Sophie I. Owen (1D1* , J. Kenneth Baillie2, Lisa Baldwin1, \\ Lottie Brown1, Rachel L. Byrne (D1), Ana I. Cubas-Atienzar (D1, Luis E. Cuevas, \\ Alice J. Fraser ${ }^{1}$, Thomas Fletcher ${ }^{3,4}$, Lynsey Goodwin ${ }^{5}$, Grant A. Kay', \\ ISARIC4C Investigators, Konstantina Kontogianni (D)1, Jenifer Mason6, \\ Peter J.M. Openshaw7 ${ }^{7}$, Stefanie Menzies ${ }^{1}$, Shona C. Moore ${ }^{5}$, \\ Malcolm G. Semple (iD) ${ }^{5}$, Joseph Taylor ${ }^{6}$, Lance C.W. Turtle (iD), \\ Christopher T. Williams ${ }^{1}$, Emily R. Adams ${ }^{1}$ \\ ${ }^{1}$ Centre for Drugs and Diagnostics Research, Liverpool School of Tropical Medicine, Liverpool, UK \\ ${ }^{2}$ Genetics and Genomics, Roslin Institute, University of Edinburgh, Edinburgh, UK \\ ${ }^{3}$ Tropical and Infectious Diseases Unit, Royal Liverpool University Hospital, Liverpool, UK \\ ${ }^{4}$ Clinical Sciences, Liverpool School of Tropical Medicine, Liverpool, UK \\ ${ }^{5}$ National Institute for Health Research (NIHR) Health Protection Research Unit (HPRU) in Emerging and Zoonotic Infections, \\ University of Liverpool, Liverpool, UK \\ ${ }^{6}$ Liverpool Clinical Laboratories, Liverpool University Hospital NHS Foundation Trust, Liverpool, UK \\ ${ }^{7}$ National Heart and Lung Institute, Imperial College London, London, UK \\ * Equal contributors
}

V1 First published: 01 Feb 2021, 6:18

https://doi.org/10.12688/wellcomeopenres.16522.1

Latest published: $01 \mathrm{Feb} 2021,6: 18$

https://doi.org/10.12688/wellcomeopenres.16522.1

\section{Abstract}

Background: Rapid mobilisation from industry and academia following the outbreak of the novel coronavirus, severe acute respiratory syndrome coronavirus 2 (SARS-CoV-2), led to the development and availability of SARS-CoV-2 lateral flow immunoassays (LFAs). High quality LFAs are urgently needed at the point of care to add to currently available diagnostic tools. In this study, we provide evaluation data for ten LFAs suitable for use at the point of care.

Methods: COVID-19 positive patients $(\mathrm{N}=45)$, confirmed by reverse transcription - quantitative polymerase chain reaction (RT-qPCR), were recruited through the International Severe Acute Respiratory and Emerging Infection Consortium - Coronavirus Clinical Characterisation Consortium (ISARIC4C) study. Sera collected from patients with influenza $A(\mathrm{~N}=20)$, tuberculosis $(\mathrm{N}=5)$, individuals with previous flavivirus exposure $(\mathrm{N}=21)$, and healthy sera $(\mathrm{N}=4)$, collected pre-

\section{Open Peer Review}

Approval Status AWAITING PEER REVIEW

Any reports and responses or comments on the article can be found at the end of the article. 
pandemic, were used as negative controls. Ten LFAs manufactured or distributed by ASBT Holdings Ltd, Cellex, Fortress Diagnostics,

Nantong Egens Biotechnology, Mologic, NG Biotech, Nal von Minden and Suzhou Herui BioMed Co. were evaluated.

Results: Compared to RT-qPCR, sensitivity of LFAs ranged from 87.0-

95.7\%. Specificity against pre-pandemic controls ranged between 92.0-100\%. Compared to IgG ELISA, sensitivity and specificity ranged between $90.5-100 \%$ and $93.2-100 \%$, respectively. Percentage agreement between LFAs and IgG ELISA ranged from 89.6-92.7\%. Inter-test agreement between LFAs and IgG ELISA ranged between kappa=0.792-0.854.

Conclusions: LFAs may serve as a useful tool for rapid confirmation of ongoing or previous infection in conjunction with clinical suspicion of COVID-19 in patients attending hospital. Impartial validation prior to commercial sale provides users with data that can inform best use settings.

Keywords

diagnostics, lateral flow immunoassays, SARS-CoV-2, COVID-19

This article is included in the Coronavirus

(COVID-19) collection. 


\section{Corresponding author: Emily R. Adams (emily.adams@lstmed.ac.uk)}

Author roles: Garrod G: Data Curation, Formal Analysis, Investigation, Methodology, Visualization, Writing - Original Draft Preparation, Writing - Review \& Editing; Owen SI: Data Curation, Formal Analysis, Investigation, Methodology, Visualization, Writing - Original Draft Preparation, Writing - Review \& Editing; Baillie JK: Funding Acquisition, Resources, Writing - Review \& Editing; Baldwin L: Project Administration, Writing - Review \& Editing; Brown L: Investigation, Writing - Review \& Editing; Byrne RL: Investigation, Writing - Review \& Editing; Cubas-Atienzar AI: Investigation, Writing - Review \& Editing; Cuevas LE: Funding Acquisition, Supervision, Writing - Review \& Editing; Fraser AJ: Investigation, Writing - Review \& Editing; Fletcher T: Resources; Goodwin L: Resources; Kay GA: Investigation, Writing - Review \& Editing; Kontogianni K: Investigation, Writing - Review \& Editing; Mason J: Resources, Writing - Review \& Editing; Openshaw PJM: Funding Acquisition, Resources, Writing - Review \& Editing; Menzies S: Investigation, Writing - Review \& Editing; Moore SC: Data Curation, Resources, Writing - Review \& Editing; Semple MG: Funding Acquisition, Resources, Writing - Review \& Editing; Taylor J: Resources, Writing - Review \& Editing; Turtle LCW: Funding Acquisition, Resources, Writing - Review \& Editing; Williams CT: Investigation, Writing - Review \& Editing; Adams ER: Conceptualization, Funding Acquisition, Methodology, Supervision, Writing Review \& Editing

Competing interests: LSTM are in a consortium with Mologic with funding received from Wellcome DFID. MGS has interests in Integrum Scientific LLC, Greensboro, NC, USA.

Grant information: This work is supported by grants from: a DFID/Wellcome Trust Epidemic Preparedness coronavirus grant (220764/Z/20/Z) to ERA and LEC, the National Institute for Health Research (NIHR; award CO-CIN-01), the Medical Research Council (MRC; grant MC_PC_19059), and by the NIHR Health Protection Research Unit (HPRU) in Emerging and Zoonotic Infections at University of Liverpool in partnership with Public Health England (PHE), in collaboration with Liverpool School of Tropical Medicine and the University of Oxford (award 200907), NIHR HPRU in Respiratory Infections at Imperial College London with PHE (award 200927), Wellcome Trust and Department for International Development (DID; 215091/Z/18/Z), the Bill and Melinda Gates Foundation (OPP1209135), Liverpool Experimental Cancer Medicine Centre (grant reference C18616/A25153), NIHR Biomedical Research Centre at Imperial College London (IS-BRC-1215-20013), and NIHR Clinical Research Network for providing infrastructure support for this research. The views expressed are those of the authors and not necessarily those of the Department of Health and Social Care, DID, NIHR, MRC, Wellcome Trust, or PHE. The funders had no role in study design, data collection and analysis, decision to publish, or preparation of the manuscript.

Copyright: $\odot 2021$ Garrod G et al. This is an open access article distributed under the terms of the Creative Commons Attribution License, which permits unrestricted use, distribution, and reproduction in any medium, provided the original work is properly cited.

How to cite this article: Garrod G, Owen SI, Baillie JK et al. Comparative evaluation of ten lateral flow immunoassays to detect SARS-CoV-2 antibodies [version 1; peer review: awaiting peer review] Wellcome Open Research 2021, 6:18

https://doi.org/10.12688/wellcomeopenres.16522.1

First published: 01 Feb 2021, 6:18 https://doi.org/10.12688/wellcomeopenres.16522.1 


\section{Introduction}

In December 2019, an outbreak of severe acute respiratory syndrome coronavirus 2 (SARS-CoV-2) emerged in Wuhan, China before spreading globally, with the World Health Organization (WHO) declaring its pandemic status in March 20201. The reference standard for diagnosis of SARS-CoV-2 are reverse transcription - quantitative polymerase chain reaction (RT-qPCR) assays. However, although a successful RT-qPCR amplification confirms an infection, the peak viral load is short lived and often occurs prior to or in the initial days after symptom onset and therefore the timing of the test is crucial ${ }^{2,3}$.

Patients tested after several days of illness may already have decreasing viral loads and negative RT-qPCR results and other markers of infection, such as immunoglobulins (Ig) could play an adjunct role in diagnosis, particularly in cases presenting $\geq 10$ days from onset of symptoms ${ }^{4}$. SARS-CoV-2 IgG and IgM can be detected in over $90 \%$ and $95 \%$ of hospitalised patients with confirmed infections respectively, with Ig presenting with simultaneous or sequential conversion ${ }^{5,6}$. Immunoglobulins are usually well established after 21 days of infection, but levels can be detectable at earlier timepoints ${ }^{7,8}$.

Global mobilisation in response to the pandemic resulted in the rapid development of lateral flow immunoassays (LFAs) for SARS-CoV-2. These assays can detect IgG, IgM, and occasionally $\operatorname{IgA}$ antibodies, are relatively simple to use and generate results in 10-15 minutes, making them appropriate for the point of care. LFAs identify individuals who have formerly experienced infections (with or without symptoms), to document the prevalence of infection in the population. Moreover, LFAs could also complement the information generated by RT-qPCR for the diagnosis of patients with presumptive COVID-19, detecting an early rise of antibodies at the time that viral loads have become undetectable.

Most LFAs are evaluated in-house by the manufacturer. However, the WHO recommends these evaluations should be complemented by independent evaluations of diagnostic accuracy. Here, we report an evaluation of ten SARS-COV-2 LFAs in a cohort of patients with RT-qPCR confirmed SARS-CoV-2 infections; in hospitalised patients with other conditions, and in healthy individuals.

\section{Methods}

\section{Ethics statement}

Research samples were provided with written consent. The study was based on samples collected for the International Severe Acute Respiratory and Emerging Infection Consortium (ISARIC) - Clinical Characterisation Consortium UK study. ISARIC CCP-UK is a prospective study based in 309 hospitals in England, Scotland, and Wales. The protocols, case report forms and details of its Independent Data and Material Access Committee are available online. Ethical approval for ISARIC CCP-UK was given by the South Central - Oxford C Research Ethics Committee in England (Ref 13/SC/0149), the Scotland A Research Ethics Committee (Ref 20/SS/0028), and the WHO
Ethics Review Committee (RPC571 and RPC572, 25th April 2013). The study protocol is available at http://isaric4c.net/protocols; study registry https://www.isrctn.com/ISRCTN66726260 and at the ISARIC4C website (https://isaric4c.net/).

\section{Participants}

Forty-five participants with RT-qPCR-confirmed SARS-CoV-2 infections admitted to National Health Service (NHS) hospitals in the UK, were recruited through the ISARIC4C study. RTqPCRs were run by UK National Health Service (NHS) accredited laboratories. Patient sera were collected $\geq 4$ days post-symptom onset and one patient provided samples at two time points. Serum samples from a further 50 patients with influenza A $(\mathrm{N}=20)$, tuberculosis $(\mathrm{N}=5)$, documented previous flavivirus exposure through vaccination or infection $(\mathrm{N}=21)$ or healthy sera $(\mathrm{N}=4)$ that had been collected pre-pandemic were included as controls.

\section{IgG enzyme-linked immunosorbent assay (ELISA)}

All samples were screened by ELISA for the detection of anti-SARS-CoV-2 IgG (Omega Diagnostics, Alva, UK), as per the manufacturer's instructions.

\section{LFAs evaluated}

All LFAs were evaluated using patient sera and compared to ELISA results. The LFAs included IgG/IgM RDT 1 (ASBT Holdings Ltd, Norfolk, UK), IgG/IgM RDT 2 (ASBT Holdings Ltd, Norfolk, UK), COVID-19 Total Ab Device (Fortress Diagnostics, Antrim, UK), Rapid COVID-19 IgG/IgM Antibody Test (Suzhou Herui BioMed Co., Ltd, China), NADAL COVID-19 IgG/IgM rapid test (Nal von Minden, Moers, Germany), qSARS-CoV-2 IgG/IgM Rapid Test (Cellex, Durham, NC, USA), COVID-19 IgG/IgM Rapid Test (Nantong Egens Biotechnology, Nantong, China), NG-Test IgG-IgM COVID19 (NG Biotech, Guipry, France), Generation one RDT prototype (Mologic, Bedfordshire, UK), and Triple Antibody RDT (Mologic, Bedfordshire, UK). All tests detect anti-SARS-CoV$2 \mathrm{IgG}$ and $\operatorname{IgM}$, except the Mologic LFAs which additionally detect anti-SARS-CoV-2 IgA. Serum samples for the evaluation had been stored at $-80^{\circ} \mathrm{C}$ and were thawed to room temperature before use. LFAs were run at room temperature according to the manufacturer's instructions, as summarised in Table 1. In brief, 5-10 $\mu$ l serum were added to the LFA well, followed by $70-100 \mu$ of the proprietary test diluent in the same or a separate buffer well. Test lines were scored as positive or negative by two independent readers at the specified reading time. The readers were blinded to each other's results and discrepant scores were resolved by a third reader.

\section{Statistical analysis}

Data analysis was carried out in Microsoft Excel (version 2012). Sensitivity was calculated as the proportion of positive tests in convalescent sera of patients with RT-qPCR confirmed infections. Specificity was calculated as the proportion of negative tests among SARS-CoV-2 negative controls collected pre-pandemic. Further calculations of sensitivity and specificity were made when using the IgG ELISA as the reference standard. The levels of agreement were calculated using Cohen's Kappa statistic ${ }^{9}$. 
Table 1. Sample and condition requirements for the ten LFAs evaluated.

\begin{tabular}{|c|c|c|c|}
\hline Test & $\begin{array}{c}\text { Sample } \\
\text { volume } \\
\text { serum }(\mu l)\end{array}$ & $\begin{array}{l}\text { Buffer } \\
\text { volume }\end{array}$ & $\begin{array}{l}\text { Time to } \\
\text { read } \\
\text { (mins) }\end{array}$ \\
\hline IgG/IgM RDT 1 (ASBT Holdings Ltd) & 10 & $80 \mu \mathrm{l}$ & 10 \\
\hline IgG/IgM RDT 2 (ASBT Holdings Ltd) & 10 & $100 \mu l$ & 10 \\
\hline COVID-19 Total Ab Device (Fortress Diagnostics) & 5 & 2 drops & 10 \\
\hline $\begin{array}{l}\text { Rapid COVID-19 IgG/IgM Antibody Test (Suzhou Herui BioMed } \\
\text { Co., Ltd, China) }\end{array}$ & 10 & $70 \mu \mathrm{l}$ & 10 \\
\hline NADAL $®$ COVID-19 IgG/IgM rapid test (Nal von Minden) & 10 & 2 drops & 10 \\
\hline qSARS-CoV-2 IgG/IgM Rapid Test (Cellex) & 10 & 2 drops & $15-20$ \\
\hline Covid-19 IgG/IgM Rapid Test (Nantong Egens Biotechnology) & 10 & 2 drops & 10 \\
\hline NG-Test $®$ IgG-IgM COVID-19 (NG Biotech) & 10 & 2 drops & 15 \\
\hline Generation one RDT prototype (Mologic) & 5 & $80 \mu l$ & 10 \\
\hline Triple Antibody RDT (Mologic) & 5 & $80 u l$ & 10 \\
\hline
\end{tabular}

\section{Results}

The 45 participants with confirmed SARS-CoV-2 infections had a median age of 58 years (IQR: 19) and $25(56 \%)$ were male. Sensitivity of the LFAs ranged from $87 \%$ to $96 \%$, with IgG ranging from $83 \%$ to $94 \%$ and $\operatorname{IgM}$ between $24 \%$ and $96 \%$. Specificity ranged from $92 \%$ to $100 \%$ for both $\operatorname{IgG}$ and $\operatorname{IgM}$ (Table 2). Differences in sensitivity and specificity across the tests were not statistically different. Sensitivity of the LFAs up to 3 weeks post-symptom onset ranged from $85 \%$ to $91 \%$ and from $83 \%$ to $100 \%$ after 3 weeks post-symptom onset. All tests except Generation one RDT prototype (Mologic, Bedfordshire, UK) recorded higher sensitivity over 3 weeks post-symptom onset (Extended data: Table $\mathrm{S}^{10}{ }^{10}$.

Forty-two (91\%) of the 45 participants with qPCR confirmed SARS-CoV-2 infections were IgG ELISA positive and $44(88 \%)$ of the 50 controls were IgG ELISA negative. Using ELISA as the reference, LFA IgG sensitivity ranged from 91\% [95\% CI: 77-97\%] to $100 \%$ [95\% CI: 92-100\%] and specificity from 93\% [95\% CI: $81-99 \%$ ] to $100 \%$ [95\% CI: 92-100\%] (Extended data: Table $\left.\mathrm{S} 2{ }^{10}\right)$. Differences across the tests were not statistically different. The percentage agreement between LFAs and ELISA ranged from $90 \%$ to $93 \%$, as shown in Table 3 . The greatest agreement between ELISA and a LFA was seen with the IgG/ IgM RDT 1 (ASBT Holdings Ltd), COVID-19 Total Ab Device (Fortress Diagnostics) and Rapid COVID-19 IgG/IgM Antibody Test (Suzhou Herui BioMed Co., Ltd) (kappa=0.854 for all three, corresponding to very good agreement).

\section{Discussion}

LFAs are potential tools for disease surveillance and the assessment of presence of antibodies to infection, which are rapid and easily conducted. In this study, we evaluated ten LFAs using sera from RT-qPCR confirmed SARS-CoV-2 infections and sera collected pre-pandemic. Although differences between tests were not statistically significant, the Fortress Total Ab Device had the highest overall sensitivity when compared to RT-qPCR. Across all tests, IgM had the widest sensitivity range (from $26 \%$ to 96\%) while IgG LFAs had similar ranges between $83 \%$ and $94 \%$. Specificity was high across all assays and four out of the ten LFAs had specificity $\geq 98 \%$. The IgG ELISA's sensitivity and specificity (Omega, UK) are 95\% ( $\geq 7$ days) and 97\% ( $\geq 10$ days) postdiagnosis, respectively ${ }^{11}$ and thus, as expected, the sensitivity and specificity of the LFAs was higher when ELISA was considered the reference standard. All LFAs had very high agreement with IgG ELISA. These data suggest LFAs can provide valuable data that is highly correlated to ELISAs, with an acknowledged small loss in sensitivity. LFAs have the advantage of being rapid and simple to run with no requirement for a laboratory or trained operators. This makes them highly suitable for low resource settings, self-testing, surveillance of the prevalence of infection or when rapid screening is required.

LFAs should have high specificity, especially in settings where infection rates are low, to avoid high numbers of false positives ${ }^{12}$. COVID-19 clinical presentation may be indistinguishable from other respiratory illnesses and LFAs could complement the information generated by RT-qPCR assays, with the tests combined identifying a larger number of individuals with current and previous SARS-CoV-2 infections. Moreover, with seasonal influenza likely coinciding with high COVID-19 incidence, these assays could play a significant role to differentiate SARS-CoV-2 from other viral infections and facilitate more targeted strategies for the management and quarantining of symptomatic patients. 
Table 2. Sensitivity and specificity of the LFAs. Sera from RT-qPCR-positive patients and a control panel of influenza A (N=20), TB $(\mathrm{N}=5)$, previous flavivirus exposure through vaccination or infection $(\mathrm{N}=21)$, and healthy sera $(\mathrm{N}=4)$, collected pre-pandemic.

\begin{tabular}{|c|c|c|c|c|c|}
\hline & Ig & $\begin{array}{l}\text { RT-qPCR SARS- } \\
\text { Cov-2 positive } \\
(N=46)\end{array}$ & $\begin{array}{l}\text { Negative } \\
\text { controls }(N=50)\end{array}$ & $\begin{array}{l}\text { Sensitivity (\%) } \\
{[95 \% \mathrm{CI}]}\end{array}$ & $\begin{array}{c}\text { Specificity (\%) } \\
\text { [95\% CI] }\end{array}$ \\
\hline \multirow[t]{3}{*}{ IgG/IgM RDT 1 (ASBT Holdings Ltd) } & $\mathbf{G}+\mathbf{M}$ & 43 & 50 & 94 [82-99] & $100[93-100]$ \\
\hline & G & 43 & 50 & 94 [82-99] & $100[93-100]$ \\
\hline & M & 17 & 50 & 37 [23- 53] & $100[93-100]$ \\
\hline \multirow[t]{3}{*}{ IgG/IgM RDT 2 (ASBT Holdings Ltd) } & $\mathbf{G}+\mathbf{M}$ & 43 & 47 & 94 [82-99] & 94 [84-99] \\
\hline & G & 41 & 50 & $89[76-96]$ & $100[93-100]$ \\
\hline & M & 43 & 47 & 94 [82-99] & 94 [84-99] \\
\hline \multirow{3}{*}{$\begin{array}{l}\text { COVID-19 Total Ab Device (Fortress } \\
\text { Diagnostics) }\end{array}$} & $\mathbf{G}+\mathbf{M}$ & 44 & 48 & $96[85-100]$ & $96[86-100]$ \\
\hline & G & 43 & 50 & 94 [82-99] & 100 [93-100] \\
\hline & M & 44 & 48 & $96[85-100]$ & $96[86-100]$ \\
\hline \multirow{3}{*}{$\begin{array}{l}\text { Rapid COVID-19 IgG/IgM Antibody Test } \\
\text { (Suzhou Herui BioMed Co., Ltd, China) }\end{array}$} & $\mathbf{G}+\mathbf{M}$ & 42 & 49 & 91 [79-98] & 98 [89-100] \\
\hline & G & 42 & 49 & 91 [79- 98] & 98 [89-100] \\
\hline & M & 12 & 50 & $26[14-41]$ & $100[93-100]$ \\
\hline \multirow{3}{*}{$\begin{array}{l}\text { NADAL } ® \text { COVID-19 IgG/IgM rapid test } \\
\text { (Nal von Minden) }\end{array}$} & $\mathbf{G}+\mathbf{M}$ & 43 & 48 & 94 [82-99] & 96 [86-100] \\
\hline & G & 40 & 50 & $87[74-95]$ & $100[93-100]$ \\
\hline & $\mathbf{M}$ & 43 & 48 & 94 [82-99] & $96[86-100]$ \\
\hline \multirow[t]{3}{*}{ qSARS-CoV-2 IgG/IgM Rapid Test (Cellex) } & $\mathbf{G}+\mathbf{M}$ & 43 & 49 & 94 [82-99] & $98[89-100]$ \\
\hline & G & 43 & 49 & 94 [82-99] & $98[89-100]$ \\
\hline & M & 11 & 50 & 24 [13-39] & 100 [93-100] \\
\hline \multirow{3}{*}{$\begin{array}{l}\text { Covid-19 IgG/IgM Rapid Test (Nantong } \\
\text { Egens Biotechnology) }\end{array}$} & $\mathbf{G}+\mathbf{M}$ & 43 & 46 & 94 [82-99] & 92 [81-98] \\
\hline & G & 43 & 46 & 94 [82- 99] & 92 [81-98] \\
\hline & $M$ & 41 & 46 & 89 [76- 96] & 92 [81-98] \\
\hline \multirow{3}{*}{$\begin{array}{l}\text { NG-Test }{ }^{\circledR} \text { IgG-IgM COVID-19 (NG } \\
\text { Biotech) }\end{array}$} & $\mathbf{G}+\mathbf{M}$ & 41 & 47 & $89[76-96]$ & 94 [84-99] \\
\hline & G & 41 & 50 & $89[76-96]$ & $100[93-100]$ \\
\hline & $\mathbf{M}$ & 41 & 47 & $89[76-96]$ & 94 [84- 99] \\
\hline \multirow{4}{*}{$\begin{array}{l}\text { Generation one RDT prototype } \\
\text { (Mologic) }\end{array}$} & $A+G+M$ & 40 & & 87 [74-95] & $100[93-100]$ \\
\hline & A & 16 & 50 & 35 [21- 50] & 100 [93-100] \\
\hline & G & 24 & 50 & 83 [69-92] & 100 [93-100] \\
\hline & $M$ & 38 & 50 & 52 [37- 67] & 100 [93-100] \\
\hline \multirow[t]{4}{*}{ Triple Antibody RDT (Mologic) } & $A+G+M$ & 42 & 48 & 94 [82-99] & 96 [86-100] \\
\hline & A & 35 & 50 & 78 [63-89] & $100[93-100]$ \\
\hline & G & 28 & 48 & 93 [82-99] & 96 [86-100] \\
\hline & $M$ & 42 & 48 & 62 [47-76] & 96 [86-100] \\
\hline
\end{tabular}


Table 3. Agreement and Cohen's kappa of LFAs and ELISA IgG.

\begin{tabular}{|c|c|c|c|c|}
\hline & \multirow[t]{2}{*}{ LFA } & \multicolumn{2}{|c|}{ ELISA } & \multirow{2}{*}{$\begin{array}{l}\text { Agreement (\%) and Kappa } \\
{[95 \% \mathrm{CI}]}\end{array}$} \\
\hline & & Positive & Negative & \\
\hline \multirow[t]{2}{*}{ IgG/IgM RDT 1 (ASBT Holdings Ltd) } & Positive & 42 & 1 & \multirow{2}{*}{$93 \%$ and 0.854 [0.751 to 0.958} \\
\hline & Negative & 6 & 47 & \\
\hline \multirow[t]{2}{*}{ IgG/IgM RDT 2 (ASBT Holdings Ltd) } & Positive & 41 & 0 & \multirow{2}{*}{$93 \%$ and 0.854 [0.751 to 0.958} \\
\hline & Negative & 7 & 48 & \\
\hline \multirow{2}{*}{$\begin{array}{l}\text { COVID-19 Total Ab Device (Fortress } \\
\text { Diagnostics) }\end{array}$} & Positive & 39 & 1 & \multirow{2}{*}{$90 \%$ and 0.792 [0.671 to 0.912$]$} \\
\hline & Negative & 9 & 47 & \\
\hline \multirow{2}{*}{$\begin{array}{l}\text { Rapid COVID-19 IgG/IgM Antibody Test (Suzhou } \\
\text { Herui BioMed Co., Ltd, China) }\end{array}$} & Positive & 42 & 1 & \multirow{2}{*}{$93 \%$ and 0.854 [0.751 to 0.958$]$} \\
\hline & Negative & 6 & 47 & \\
\hline \multirow{2}{*}{$\begin{array}{l}\text { NADAL } ® \text { COVID-19 IgG/IgM rapid test (Nal von } \\
\text { Minden) }\end{array}$} & Positive & 39 & 1 & \multirow{2}{*}{$90 \%$ and 0.792 [0.671 to 0.912 . } \\
\hline & Negative & 9 & 47 & \\
\hline \multirow[t]{2}{*}{ qSARS-CoV-2 IgG/IgM Rapid Test (Cellex) } & Positive & 42 & 2 & \multirow{2}{*}{$92 \%$ and 0.833 [0.723 to 0.944$]$} \\
\hline & Negative & 6 & 46 & \\
\hline \multirow{2}{*}{$\begin{array}{l}\text { Covid-19 IgG/IgM Rapid Test (Nantong Egens } \\
\text { Biotechnology) }\end{array}$} & Positive & 42 & 4 & \multirow{2}{*}{$80 \%$ and 0.792 [0.670 to 0.914$]$} \\
\hline & Negative & 6 & 44 & \\
\hline \multirow[t]{2}{*}{ NG-Test ${ }^{\circledR}$ IgG-IgM COVID-19 (NG Biotech) } & Positive & 41 & 1 & \multirow{2}{*}{$91 \%$ and 0.813 [0.698 to 0.928$]$} \\
\hline & Negative & 8 & 46 & \\
\hline \multirow[t]{2}{*}{ Generation one RDT prototype (Mologic) } & Positive & 38 & 0 & \multirow{2}{*}{$90 \%$ and 0.792 [0.672 to 0.911$]$} \\
\hline & Negative & 10 & 48 & \\
\hline \multirow[t]{2}{*}{ Triple Antibody RDT (Mologic) } & Positive & 42 & 2 & \multirow{2}{*}{$92 \%$ and 0.832 [0.721 to 0.943] } \\
\hline & Negative & 6 & 45 & \\
\hline
\end{tabular}

We acknowledge this validation has several limitations, as it included a small sample size, without enough power to find small differences in the performance of the assays, and therefore our findings can only be shown to be indicative of the likely findings of appropriately powered evaluations. All SARS-CoV-2 positive samples were obtained from hospitalised patients as a marker of severe COVID-19 who may be expected to have high levels of antibodies, and our findings need to be replicated in asymptomatic individuals and in patients presenting with mild symptoms and in community settings. Furthermore, all testing was run under laboratory conditions by laboratory staff and the results may vary when conducted at the point of need and self-testing by untrained individuals. Finally, we were constrained to use serum, and further evaluations are needed to evaluate their performance on whole venous or capillary blood. Test performance on capillary blood, including a comparison of self-testing and laboratory testing, would inform the potential for use of less invasive sample collection methods.
Validation of test performance in people with presumptive SARS-COV-2 infection who are asymptomatic or are experiencing mild infection is of particular interest as reports indicate a large proportion of individuals testing positive by RT-PCR have no symptoms on the day of sampling, a week prior and a week after a positive result ${ }^{13}$. LFAs ease of use and their fast time to results lends them to self-testing outside a clinical or laboratory environment. However, validation of test performance under these more challenging and less controlled environments is needed. Further evaluations of LFAs should include other coronaviruses and respiratory illnesses with overlapping signs and symptoms of COVID-19, including other causes of pneumonia, upper and lower respiratory infection, rhinoviruses, respiratory syncytial virus and influenza.

The impartial evaluation of LFAs, as recommended by the WHO, can confirm the evaluations of the developer's validation data, and inform best-use settings. LFAs are a valuable tool which 
could be applied at the point of need in conjunction with other assays to provide a more holistic diagnosis and to monitor the prevalence of infection.

\section{Data availability}

\section{Underlying data}

Dryad: Comparative evaluation of ten lateral flow immunoassays to detect SARS-CoV-2 antibodies, doi.org/10.5061/dryad. brv $15 \mathrm{dv} 8 \mathrm{~h}^{10}$.

\section{Extended data}

Dryad: Comparative evaluation of ten lateral flow immunoassays to detect SARS-CoV-2 antibodies, doi.org/10.5061/dryad. brv $15 \mathrm{dv} 8 \mathrm{~h}^{10}$.

This project contains the following extended data:

- Table S1. Sensitivity of LFAs in RT-qPCR-positive samples $\leq 21$ days $(\mathrm{N}=33)$ and $>21$ days post-symptom onset $(\mathrm{N}=12)$.

- Table S2. Sensitivity and specificity of the LFAs in comparison to IgG ELISA. Anti-SARS-CoV-2 ELISAIgG positive $(\mathrm{N}=42)$ and anti-SARS-CoV-2 ELISA-IgG negative samples (influenza $\mathrm{A}, \mathrm{N}=18, \mathrm{~TB}, \mathrm{~N}=3$, flavivirus exposure, $\mathrm{N}=21$, and healthy controls, $\mathrm{N}=4$ ).

Data are available under the terms of the Creative Commons Zero "No rights reserved" data waiver (CCO 1.0 Public domain dedication)

\section{Acknowledgements}

\section{ISARIC4C Investigators}

Consortium Lead Investigator: J Kenneth Baillie, Chief Investigator: Malcolm G Semple, Co-Lead Investigator: Peter JM Openshaw. ISARIC Clinical Coordinator: Gail Carson. Co-Investigators: Beatrice Alex, Benjamin Bach, Wendy S Barclay, Debby Bogaert, Meera Chand, Graham S Cooke, Annemarie B Docherty, Jake Dunning, Ana da Silva Filipe, Tom Fletcher, Christopher A Green, Ewen M Harrison, Julian A Hiscox, Antonia Ying Wai Ho, Peter W Horby, Samreen Ijaz, Saye Khoo, Paul Klenerman, Andrew Law, Wei Shen Lim, Alexander J Mentzer, Laura Merson, Alison M Meynert, Mahdad Noursadeghi, Shona C Moore, Massimo Palmarini, William A Paxton, Georgios Pollakis, Nicholas Price, Andrew Rambaut, David L Robertson, Clark D Russell, Vanessa Sancho-Shimizu, Janet $\mathrm{T}$ Scott, Thushan de Silva, Louise Sigfrid, Tom Solomon, Shiranee Sriskandan, David Stuart, Charlotte Summers, Richard S Tedder, Emma C Thomson, AA Roger Thompson, Ryan S Thwaites, Lance CW Turtle, Maria Zambon. Project Managers: Hayley Hardwick, Chloe Donohue, Ruth Lyons, Fiona Griffiths, Wilna Oosthuyzen. Data Analysts: Lisa Norman, Riinu Pius, Tom M Drake, Cameron J Fairfield, Stephen Knight, Kenneth A Mclean, Derek Murphy, Catherine A Shaw. Data and Information System Managers: Jo Dalton, James Lee, Daniel Plotkin, Michelle Girvan, Egle Saviciute, Stephanie Roberts, Janet Harrison, Laura Marsh, Marie Connor, Sophie Halpin, Clare Jackson, Carrol
Gamble. Data integration and presentation: Gary Leeming, Andrew Law, Murray Wham, Sara Clohisey, Ross Hendry, James Scott-Brown. Material Management: William Greenhalf, Victoria Shaw, Sarah McDonald. Patient engagement: Seán Keating Outbreak Laboratory Staff and Volunteers: Katie A. Ahmed, Jane A Armstrong, Milton Ashworth, Innocent G Asiimwe, Siddharth Bakshi, Samantha L Barlow, Laura Booth, Benjamin Brennan, Katie Bullock, Benjamin WA Catterall, Jordan J Clark, Emily A Clarke, Sarah Cole, Louise Cooper, Helen Cox, Christopher Davis, Oslem Dincarslan, Chris Dunn, Philip Dyer, Angela Elliott, Anthony Evans, Lorna Finch, Lewis WS Fisher, Terry Foster, Isabel Garcia-Dorival, Willliam Greenhalf, Philip Gunning, Catherine Hartley, Antonia Ho, Rebecca L Jensen, Christopher B Jones, Trevor R Jones, Shadia Khandaker, Katharine King, Robyn T. Kiy, Chrysa Koukorava, Annette Lake, Suzannah Lant, Diane Latawiec, L Lavelle-Langham, Daniella Lefteri, Lauren Lett, Lucia A Livoti, Maria Mancini, Sarah McDonald, Laurence McEvoy, John McLauchlan, Soeren Metelmann, Nahida S Miah, Joanna Middleton, Joyce Mitchell, Shona C Moore, Ellen G Murphy, Rebekah Penrice-Randal, Jack Pilgrim, Tessa Prince, Will Reynolds, P. Matthew Ridley, Debby Sales, Victoria E Shaw, Rebecca K Shears, Benjamin Small, Krishanthi S Subramaniam, Agnieska Szemiel, Aislynn Taggart, Jolanta Tanianis-Hughes, Jordan Thomas, Erwan Trochu, Libby van Tonder, Eve Wilcock, J. Eunice Zhang. Local Principal Investigators: Kayode Adeniji, Daniel Agranoff, Ken Agwuh, Dhiraj Ail, Ana Alegria, Brian Angus, Abdul Ashish, Dougal Atkinson, Shahedal Bari, Gavin Barlow, Stella Barnass, Nicholas Barrett, Christopher Bassford, David Baxter, Michael Beadsworth, Jolanta Bernatoniene, John Berridge, Nicola Best, Pieter Bothma, David Brealey, Robin Brittain-Long, Naomi Bulteel, Tom Burden, Andrew Burtenshaw, Vikki Caruth, David Chadwick, Duncan Chambler, Nigel Chee, Jenny Child, Srikanth Chukkambotla, Tom Clark, Paul Collini, Catherine Cosgrove, Jason Cupitt, Maria-Teresa Cutino-Moguel, Paul Dark, Chris Dawson, Samir Dervisevic, Phil Donnison, Sam Douthwaite, Ingrid DuRand, Ahilanadan Dushianthan, Tristan Dyer, Cariad Evans, Chi Eziefula, Chrisopher Fegan, Adam Finn, Duncan Fullerton, Sanjeev Garg, Sanjeev Garg, Atul Garg, Effrossyni GkraniaKlotsas, Jo Godden, Arthur Goldsmith, Clive Graham, Elaine Hardy, Stuart Hartshorn, Daniel Harvey, Peter Havalda, Daniel B Hawcutt, Maria Hobrok, Luke Hodgson, Anil Hormis, Michael Jacobs, Susan Jain, Paul Jennings, Agilan Kaliappan, Vidya Kasipandian, Stephen Kegg, Michael Kelsey, Jason Kendall, Caroline Kerrison, Ian Kerslake, Oliver Koch, Gouri Koduri, George Koshy, Shondipon Laha, Steven Laird, Susan Larkin, Tamas Leiner, Patrick Lillie, James Limb, Vanessa Linnett, Jeff Little, Michael MacMahon, Emily MacNaughton, Ravish Mankregod, Huw Masson, Elijah Matovu, Katherine McCullough, Ruth McEwen, Manjula Meda, Gary Mills, Jane Minton, Mariyam Mirfenderesky, Kavya Mohandas, Quen Mok, James Moon, Elinoor Moore, Patrick Morgan, Craig Morris, Katherine Mortimore, Samuel Moses, Mbiye Mpenge, Rohinton Mulla, Michael Murphy, Megan Nagel, Thapas Nagarajan, Mark Nelson, Igor Otahal, Mark Pais, Selva Panchatsharam, Hassan Paraiso, Brij Patel, Natalie Pattison, Justin Pepperell, Mark Peters, Mandeep Phull, Stefania Pintus, Jagtur Singh Pooni, Frank Post, David Price, Rachel Prout, Nikolas Rae, Henrik Reschreiter, Tim Reynolds, Neil Richardson, Mark Roberts, Devender Roberts, Alistair Rose, Guy 
Rousseau, Brendan Ryan, Taranprit Saluja, Aarti Shah, Prad Shanmuga, Anil Sharma, Anna Shawcross, Jeremy Sizer, Manu Shankar-Hari, Richard Smith, Catherine Snelson, Nick Spittle, Nikki Staines, Tom Stambach, Richard Stewart, Pradeep Subudhi, Tamas Szakmany, Kate Tatham, Jo Thomas, Chris Thompson, Robert Thompson, Ascanio Tridente, Darell Tupper-Carey, Mary Twagira, Andrew Ustianowski, Nick Vallotton, Lisa Vincent-Smith, Shico Visuvanathan, Alan Vuylsteke, Sam Waddy, Rachel Wake, Andrew Walden, Ingeborg Welters, Tony Whitehouse, Paul Whittaker, Ashley Whittington, Meme Wijesinghe, Martin Williams, Lawrence Wilson, Sarah
Wilson, Stephen Winchester, Martin Wiselka, Adam Wolverson, Daniel G Wooton, Andrew Workman, Bryan Yates, and Peter Young.

This work uses data provided by patients and collected by the NHS as part of their care and support \#DataSavesLives. We are extremely grateful to the 2,648 frontline NHS clinical and research staff and volunteer medical students, who collected this data in challenging circumstances; and the generosity of the participants and their families for their individual contributions in these difficult times.
1. World Health Organisation: Coronavirus Disease 2019 (COVID-19) Situation Report - 51. 2020. Reference Source

2. He X, Lau EHY, Wu P, et al.: Temporal dynamics in viral shedding and transmissibility of COVID-19. Nat Med. 2020; 26(5): 672-675. PubMed Abstract | Publisher Full Text

3. Liu $Y$, Yan $L M$, Wan $L$, et al.: Viral dynamics in mild and severe cases of COVID-19. Lancet Infect Dis. 2020; 20(6): 656-657. PubMed Abstract | Publisher Full Text | Free Full Text

4. FIND: GENERAL INFORMATION ON Ag-AND Ab-DETECTION RDTS FOR COVID-19. 2020

5. $\quad$ Long QX, Liu BZ, Deng HJ, et al.: Antibody responses to SARS-CoV-2 in patients with COVID-19. Nat Med. 2020; 26(6): 845-848. PubMed Abstract | Publisher Full Text

6. Staines HM, Kirwan DE, Clark DJ, et al.: Dynamics of IgG seroconversion and pathophysiology of COVID-19 infections. medRxiv. 2020; 2020.06.07.20124636. Publisher Full Text

7. $\mathrm{Ma} \mathrm{H}$, Zeng $\mathrm{W}$, He H, et al:: Serum IgA, IgM, and IgG responses in COVID-19. Cell Mol Immunol. 2020; 17(7): 773-775.

PubMed Abstract | Publisher Full Text | Free Full Text
8. Padoan A, Sciacovelli L, Basso D, et al.: IgA-Ab response to spike glycoprotein of SARS-CoV-2 in patients with COVID-19: A longitudinal study. Clin Chim Acta. 2020; 507: 164-166.

PubMed Abstract | Publisher Full Text | Free Full Text

9. Landis JR, Koch GG: The measurement of observer agreement for categorical data. Biometrics. 1977; 33(1): 159-74. PubMed Abstract

10. Garrod G, Owen S, Baillie K, et al.: Data for: Comparative evaluation of ten lateral flow immunoassays to detect SARS-CoV-2 antibodies. Dryad. [data collection]. 2020. http://www.doi.org/10.5061/dryad.brv15dv8h

11. Adams ER, Augustin Y, Byrne RL, et al.: Rapid development of COVID-19 rapid diagnostics for low resource settings: accelerating delivery through transparency, responsiveness, and open collaboration. medRxiv. 2020; 2020.04.29.20082099. Publisher Full Text

12. Frasier SL: False Positive Alarm. Sci Am. 2020; 323: 12-13.

13. Pouwels KB, House T, Robotham JV, et al.: Community prevalence of SARS-CoV-2 in England: Results from the ONS Coronavirus Infection Survey Pilot. medRxiv. 2020; $1-13$.

Publisher Full Text 